\title{
The Genomic Landscape of Plasma Cells in Systemic Light chain Amyloidosis
}

Authors: Eileen M. Boyle ${ }^{1,2,3}$, Cody Ashby ${ }^{1}$, Christopher P. Wardell ${ }^{1}$, Dorota Rowczenio ${ }^{4}$, Sajitha Sachchithanantham ${ }^{4}$, Yan Wang ${ }^{1}$, Sarah K. Johnson ${ }^{1}$, Michael A. Bauer ${ }^{1}$, Niels Weinhold ${ }^{1}$, Martin F. Kaiser $^{5}$, David C. Johnson ${ }^{5}$, John R. Jones ${ }^{5}$, Charlotte Pawlyn ${ }^{5}$, Paula Proszek ${ }^{5}$, Carolina Schinke ${ }^{1}$, Thierry Facon ${ }^{2}$, Charles Dumontet ${ }^{3}$, Faith E. Davies ${ }^{1}$, Gareth J. Morgan ${ }^{1}$, Brian A. Walker ${ }^{1 *}$, and Ashutosh D. Wechalekar ${ }^{4 *}$

\footnotetext{
${ }^{1}$ Myeloma Institute, University of Arkansas for Medical Sciences, Little Rock, AR, USA.

${ }^{2}$ Service des maladies du sang. Hôpital Claude Huriez, Lille University Hospital, Lille, FRANCE.

${ }^{3}$ INSERM 1052/CNRS 5286 Cancer Research Center of Lyon, Lyon, FRANCE

${ }^{4}$ National Amyloidosis Centre, University College London, London, UK

${ }^{5}$ The Institute of Cancer Research, London, UK

*These authors contributed equally
}

Corresponding Author: Ashutosh D. Wechalekar

Address: National Amyloidosis Centre, University College London (Royal Free Campus), Rowland Hill Street, London NW3 2PF, UK

Email: a.wechalekar@ucl.ac.uk

Tel: +44 (0)20 74332802

Fax: +44 (0)20 74332817

Running title: Landscape of AL mutations

Words: 1193 (1200)

Figures and tables: $1+1(2)$

References: 18 (25)

Category: Letter 


\section{TO THE EDITOR:}

The key event in the pathogenesis of Systemic light chain amyloidosis (AL) is an unstable misfolded secondary or tertiary structure of a monoclonal immunoglobulin (IG) light chain, that precipitates in the extracellular compartments. ${ }^{1}$ The plasma-cell disorder underlying AL, is likely to lie within the common spectrum of plasma-cell diseases, but limited confirmatory data analysing the genetic architecture of AL are available. ${ }^{2}$

To address this, we prospectively included twenty-four newly diagnosed histologically proven AL samples from the National Amyloid Centre, University College London, UK, prior to any treatment. AL was confirmed by immunohistochemistry or mass spectrometry. CD138+ cells and peripheral blood were isolated, DNA extracted and used in an exome capture protocol and sequenced as previously published. ${ }^{3}$ This AL dataset was compared to previously published $\mathrm{MGUS}^{4}$ and $\mathrm{MM}^{5,6}$ datasets and filtered similarly to ensure comparability of mutation numbers. Detailed methods and Patient's characteristics may be found in the Supplemental-Methods and Supplemental-Table 1.

In terms of mutational burden, the median number of exonic, non-IG, non-synonymous mutations per sample that had a tumour variant allelic fraction greater than 5\% was 39 (interquartile range (IQR): 5-185) which is more than was seen in MGUS $^{4}$ (20 (IQR: 1-41), $\mathrm{p}=0.002$ ) but not statistically different from $\mathrm{MM}^{6}$ (35 (IQR: 3-74), $\left.\mathrm{p}=0.4\right)$, Figure 1A. When analysing the individual genes, we identified a total of 1491 genes that were mutated in our dataset with 236 of them being mutated more than once. As expected, there was no unifying mutation in AL. The dataset was too small to identify any significantly mutated genes. ${ }^{7}$ Among the recurrently mutated genes (Supplemental-Table 2), mutations in $I L 7 R$, a gene that encodes for a cell surface receptor known to regulate $\mathrm{V}(\mathrm{D}) \mathrm{J}$ recombination by altering the accessibility of DNA substrates to the recombinase in pro-B-cells, ${ }^{8}$ were noted. Thirtyseven percent of samples $(n=9)$ had a mutation in one of the 63 previously described MMdriver genes. ${ }^{9}$ This is less than MM $(84.1 \%)^{9}$ and similar to MGUS $(36 \%){ }^{4}$ The number of mutated driver-genes per sample ranged from zero to five. Among the 63 driver-genes, 13 were mutated, $\underline{\text { Table 1 }}$. Most of these mutations were sub-clonal with IG-adjusted estimated tumour fractions ranging from $18 \%$ to $100 \%$ (Supplemental-Table 3). We identified cases with MM hotspot mutations in NRAS (Q61R and Q61H) but not in KRAS. Even though a trend for fewer KRAS mutations in AL was observed, there was no significant difference in the incidence of driver mutations between MM, MGUS, and AL, Figure 1B. Interestingly there were mutations in some of the other driver-genes such as EGRl (Q95R), DIS3 (D479E, 
M667L), IRF4 (S332G), and TRAF3 (K99_sp). Only the same TRAF3 (K99_sp) and DIS3 (D479E, M667L) mutations were seen in the MM dataset, Supplemental-Figure 1A-E. ${ }^{9}$ In $\mathrm{MM}$, IRF4 mutations are associated with $\mathrm{t}(11 ; 14)$, and in this AL dataset the IRF4 mutation (S332G) occurred in a non $\mathrm{t}(11 ; 14)$ patient and was neither at the K123R hotspot seen in $\mathrm{MM}^{6}$ nor the L116R seen in CLL. ${ }^{10}$ There were no mutations in the previously reported adverse prognostic genes such as ATM, ATR, ZFHX4 and TP53, nevertheless mutations in BRCA2 (P1088T, N372H) and the driver-gene EP300 (I997V) were seen suggesting DNArepair pathway involvement. Copy-number analysis did not reveal any copy number changes at these loci suggesting the absence of bi-allelic inactivation. Finally, evidence would suggest the presence of NF- $\mathrm{BB}$ pathway activation, with not only mutations in the driver-genes (such as TRAF3, IRF4) but also mutations in kinases (such as LYN (I165T)), downstream transducing molecules $(C A R D 11(\mathrm{R} 1077 \mathrm{~V}))$ and inhibitors (NFKBIE_sp), $\underline{\text { Supplemental- }}$

Figure 2. We compared the mutational landscape of AL to previously sequenced MM $(\mathrm{n}=1273)^{9}$ and MGUS $(\mathrm{n}=33)^{4}$ samples. Overall, there were 101 genes in common between AL, MGUS, and MM. Ninety-three percent of the AL-mutated genes $(n=1386)$ were shared with either MM or MGUS. Only 7\% of these genes $(n=105)$ had not previously been reported in MM or MGUS, Supplemental-Figure 3 and Table 4. None of these mutations were recurrent. A Gene enrichment analysis of the 105 genes that were mutated in AL only ${ }^{11}$ did not reveal any specific pathway enrichment suggesting they are random. There were no mutations in the ribosome sub-unit genes. ${ }^{12}$ Among the 68 differentially expressed genes (Paiva et al.), we only identified a mutation in TIAM2 previously reported in MM. ${ }^{2}$ Of note, we did identify a mutation in the $8^{\text {th }}$ exon of PSMA2 gene (P223S) that was among the differentially expressed genes found by Abraham et $\mathrm{al}^{13}$. In a hierarchical clustering approach of driver mutation based on previously published data, ${ }^{4,5,14,15}$ AL clustered with MGUS and was closely related to MM, Figure 1C. In terms of translocations, we identified seven $\mathrm{t}(11 ; 14)$ encompassing $30 \%$ of cases. The breakpoints, located 2-600 kb upstream of CCND1, were consistent with those seen in other lymphoid malignancies, Supplemental-Figure 4. They were all generated via class switch recombination with breakpoints occurring in the IGHAI (2/7) and IGHM (5/7) switch regions. There was no evidence of other canonical translocation but we identified a $\mathrm{t}(1 ; 14)$ involving $I G H G 4$ and the $R C C 1$ gene in a $\mathrm{t}(11 ; 14)$ patient, Supplemental-Figure 5. There was no evidence of inter-chromosomal translocations involving MYC; one patient had an 8q24 gain, 5' to MIR1208, suggesting MYC rearrangements also occur in AL. Regarding other cytogenetic abnormalities, there was no difference in the incidence of copy number changes with the exception of del(1p), and 
$\operatorname{del}(14 q)$ that was lower than expected in $\mathrm{MM}(\mathrm{n}=1,4 \%, \mathrm{p}=0.008$ and $\mathrm{n}=0, \mathrm{p}<0.0001$ respectively) but similar to MGUS, Supplemental-Figure 6.

This is the largest dataset of whole exome sequencing (WES) of AL to date and combined, these data suggest the underlying disease in AL resembles other plasma-cell disorders such as MGUS and MM. The number of mutations in AL was comparable to MM suggesting AL is a more complex disease than MGUS. Like previously published AL cases that had undergone $\mathrm{WES},{ }^{2}$ we failed to identify any unifying mutation. We were nonetheless able to detect MMdefined driver mutations. They occurred at similar frequencies to MGUS and were less common than in MM. They were predominantly sub-clonal suggesting they occurred late during disease progression. There was evidence of mitogen activated protein kinase (MAPK) activation with NRAS mutations (8\%) in keeping with previously published data by Rossi et $\mathrm{al}^{16}, \mathrm{NF}-\kappa \mathrm{B}$ activation (17\%) and DNA-repair pathway alterations (12\%).Unlike previous reports, none of the NRAS mutated patients had classical CRAB criteria but one had another myeloma defining event (SFLC ratio>100 with an involved chain greater than $1000 \mathrm{mg} / \mathrm{L}$ ). ${ }^{17}$ These data are consistent with observations from MGUS and SMM where NRAS mutations may be found with lower frequencies than in MM. ${ }^{4,16,18}$ There was a $93 \%$ overlap in mutated genes indicating a common spectrum of mutations between $\mathrm{AL}$ and other plasma-cell disorders. Attempts to cluster patients based on MM-driver genes placed AL close to MM and MGUS and not with other lymphoid malignancies.

Overall AL showed a similar mutation burden to MM but resembled MGUS in terms of copy number changes and driver-gene mutations suggesting $\mathrm{AL}$ lies within the continuous spectrum from MGUS to MM. Given that the similarities with MGUS and MM are outweighing the differences, it is unlikely that the plasmacell biology per se explains the clinical presentation of AL, however, these data support the ongoing use of myeloma-based therapy for this disease. 
Boyle et al.

Landscape of AL mutations

\section{Acknowledgements}

We acknowledge The Institute of Cancer Research Tumour Profiling Unit for their support. This work was supported by a Myeloma UK program grant, a Cancer Research UK CTAAC sample collection grant and a Cancer Research UK Biomarkers and Imaging Discovery and Development grant as well as funds from the National Institute of Health Biomedical Research Centre at the Royal Marsden Hospital. EMB was supported by a grant from the Fédération française de recherche sur le myélome et les gammapathies under the auspices of La Fondation de France.

Authorship contributions

Conception and design of the study: EMB, DR, BAW, GJM, ADW

Acquisition of data: EMB, DR, BAW, SS, ADW, PP

Analysis of data: EMB, CA, YW, CPW, MAB, NW, BAW, MFK, DCJ, JRJ, CP, TF, CD, CS, FED, GJM

Writing of manuscript: EMB, BAW, GJM, ADW

Review or revision of manuscript: all.

\section{Conflicts of Interest}

The authors have no relevant conflicts of interest to disclose. 


\section{References}

1. Merlini G, Bellotti V. Molecular mechanisms of amyloidosis. N. Engl. J. Med. 2003;349(6):583-596.

2. Paiva B, Martinez-Lopez J, Corchete LA, et al. Phenotypic, transcriptomic, and genomic features of clonal plasma cells in light-chain amyloidosis. Blood. 2016;127(24):3035-3039.

3. Kozarewa I, Rosa-Rosa JM, Wardell CP, et al. A Modified Method for Whole Exome Resequencing from Minimal Amounts of Starting DNA. PLOS ONE. 2012;7(3):e32617.

4. Mikulasova A, Wardell CP, Murison A, et al. The spectrum of somatic mutations in monoclonal gammopathy of undetermined significance indicates a less complex genomic landscape than that in multiple myeloma. Haematologica. 2017;102(9):1617-1625.

5. Walker BA, Boyle EM, Wardell CP, et al. Mutational Spectrum, Copy Number Changes, and Outcome: Results of a Sequencing Study of Patients With Newly Diagnosed Myeloma. J. Clin. Oncol. Off. J. Am. Soc. Clin. Oncol. 2015; Nov 20;33(33):3911-20

6. Walker BA, Mavrommatis K, Wardell CP, et al. Identification of novel mutational drivers reveals oncogene dependencies in multiple myeloma. Blood. 2018;132(6):587-597.

7. Lawrence MS, Stojanov P, Polak P, et al. Mutational heterogeneity in cancer and the search for new cancer-associated genes. Nature. 2013;499(7457):214-218.

8. Corcoran AE, Riddell A, Krooshoop D, Venkitaraman AR. Impaired immunoglobulin gene rearrangement in mice lacking the IL-7 receptor. Nature. 1998;391(6670):904-907.

9. Walker BA, Mavrommatis K, Wardell CP, et al. A high-risk, Double-Hit, group of newly diagnosed myeloma identified by genomic analysis. Leukemia. 2018; doi: 10.1038/s41375-018-0196-8.

10. Havelange V, Pekarsky Y, Nakamura T, et al. IRF4 mutations in chronic lymphocytic leukemia. Blood. 2011;118(10):2827-2829.

11. Thomas PD, Campbell MJ, Kejariwal A, et al. PANTHER: A Library of Protein Families and Subfamilies Indexed by Function. Genome Res. 2003;13(9):2129-2141.

12. Kryukov F, Kryukova E, Brozova L, et al. Does AL amyloidosis have a unique genomic profile? Gene expression profiling meta-analysis and literature overview. Gene. 2016;591(2):490-498.

13. Abraham RS, Ballman KV, Dispenzieri A, et al. Functional gene expression analysis of clonal plasma cells identifies a unique molecular profile for light chain amyloidosis. Blood. 2005;105(2):794-803.

14. Lohr JG, Stojanov P, Lawrence MS, et al. Discovery and prioritization of somatic mutations in diffuse large B-cell lymphoma (DLBCL) by whole-exome sequencing. Proc. Natl. Acad. Sci. U. S. A. 2012;109(10):3879-3884.

15. Landau DA, Sun C, Rosebrock D, et al. The evolutionary landscape of chronic lymphocytic leukemia treated with ibrutinib targeted therapy. Nat. Commun. 2017;8(1):2185.

16. Rossi A, Voigtlaender M, Janjetovic S, et al. Mutational landscape reflects the biological continuum of plasma cell dyscrasias. Blood Cancer J. 2017;7(2):e537.

17. Kim SJ, Shin H-T, Lee H-O, et al. Recurrent mutations of MAPK pathway genes in multiple myeloma but not in amyloid light-chain amyloidosis. Oncotarget. 2016;7(42):68350-68359.

18. Bezieau S, Devilder MC, Avet-Loiseau H, et al. High incidence of $\mathrm{N}$ and K-Ras activating mutations in multiple myeloma and primary plasma cell leukemia at diagnosis. Hum. Mutat. 2001;18(3):212-224. 
Boyle et al.

Tables

Table 1: Driver mutations found in $\mathbf{A L}$

\begin{tabular}{|c|c|c|c|}
\hline Gene & $\begin{array}{c}\text { Number of } \\
\text { mutations }\end{array}$ & Number of patients & Variants \\
\hline DIS3 & 2 & 1 & D479E, M667L \\
\hline DUSP2 & 1 & 1 & Splice donor variant \\
\hline EGR1 & 1 & 1 & Q95R \\
\hline EP300 & 1 & 1 & I997V \\
\hline IRF4 & 1 & 1 & S332G \\
\hline KLHL6 & 1 & 1 & V337M \\
\hline KMT2B & 1 & 1 & P204H \\
\hline NRAS & 2 & 2 & Q61R; Q61H \\
\hline TEH 2 & 1 & 1 & F363L \\
\hline TRAF3 & 1 & 1 & P363L \\
\hline ZFP36L1 & 1 & 1 & Splice donor variant \\
\hline ZNF292 & 1 & 1 & I1740V \\
\hline
\end{tabular}


Boyle et al.

Landscape of AL mutations

\section{Figure Legends}

Figure 1: The mutational spectrum of amyloidosis resembles MM and MGUS. A: Boxplot suggesting AL has more mutations than MGUS but not a statistically different number than MM. B: Frequencies of select driver mutations in AL, MGUS and MM. C: Clustering of driver-mutation frequencies 
A.

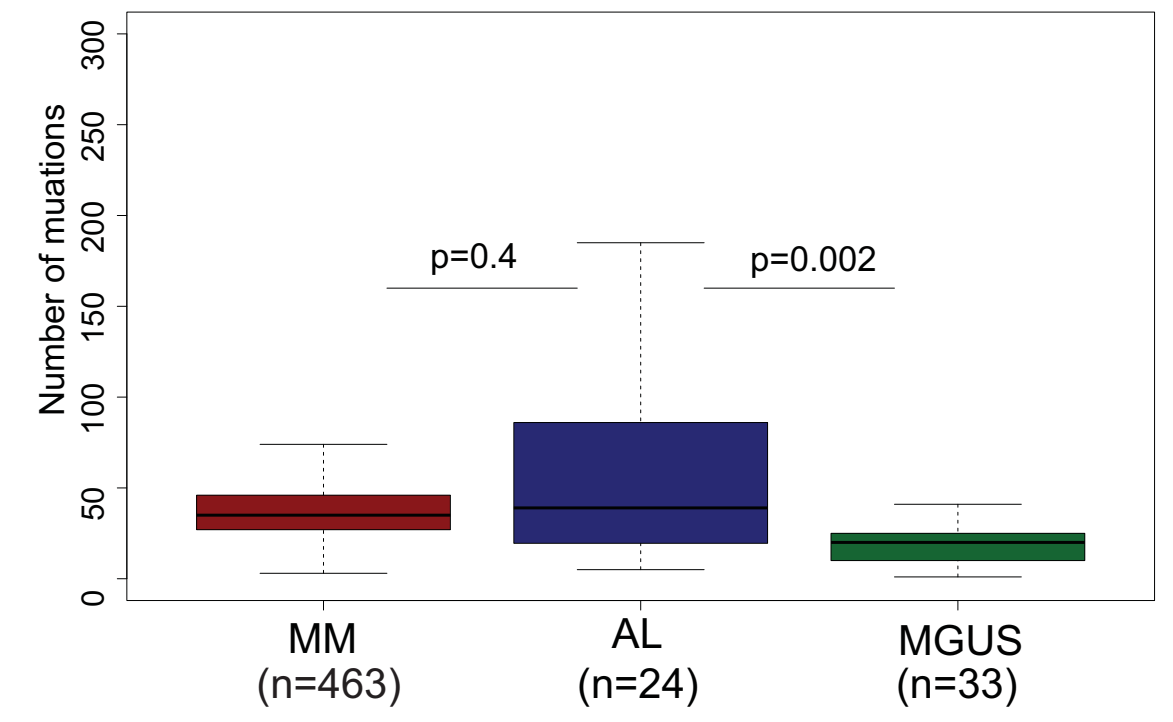

B.

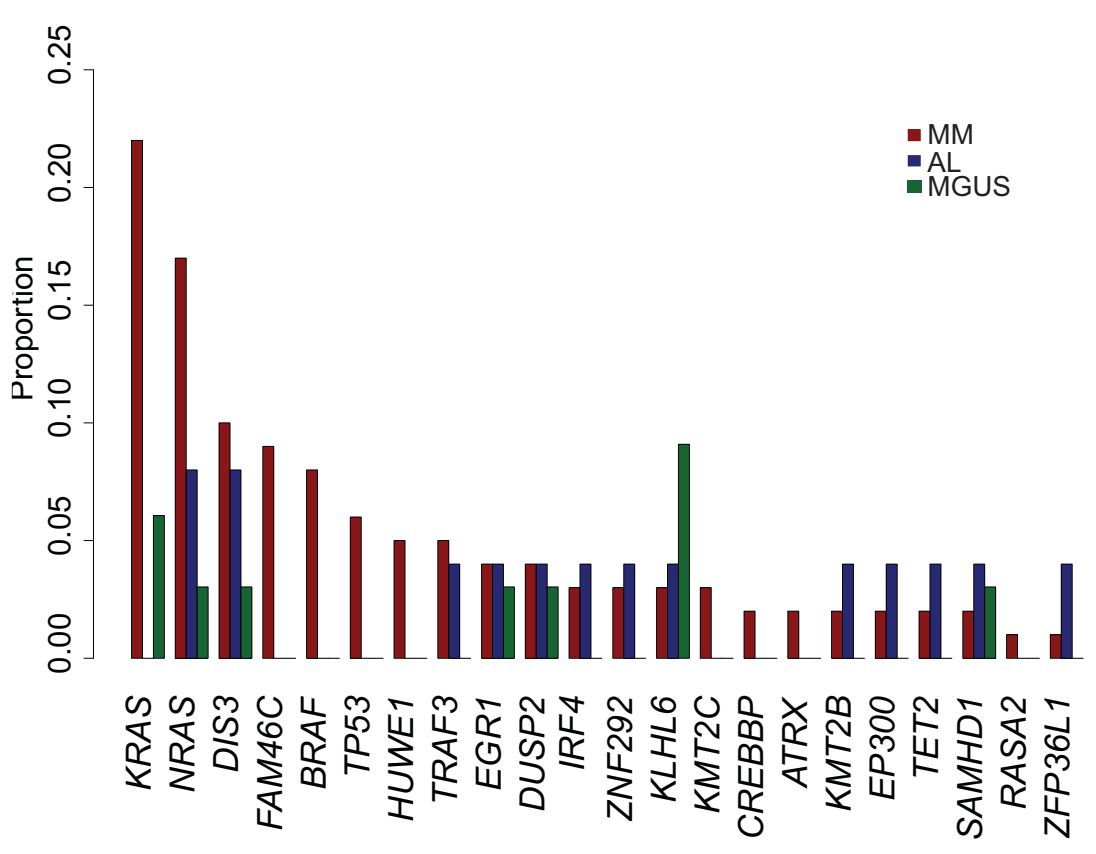

c.
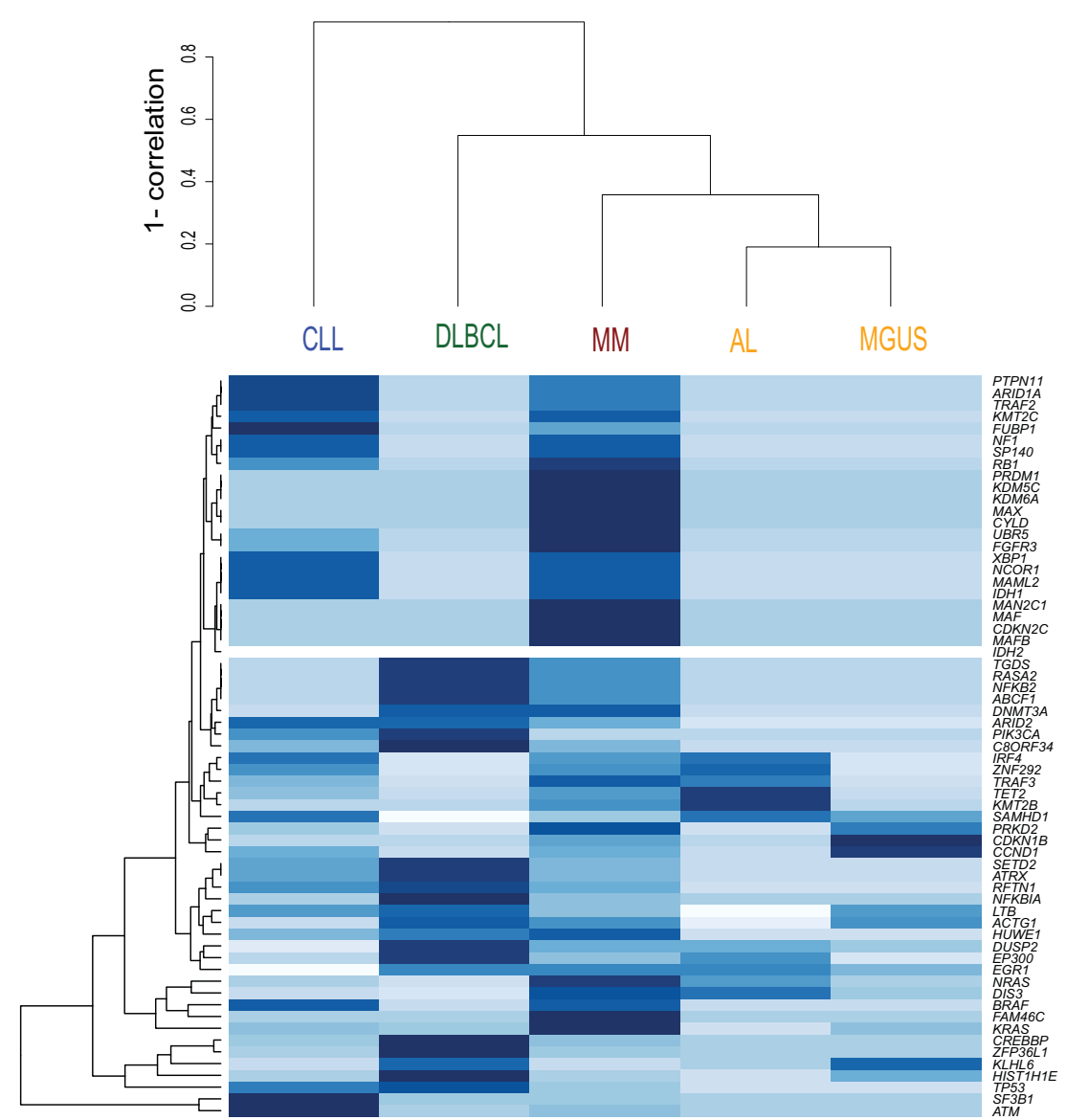


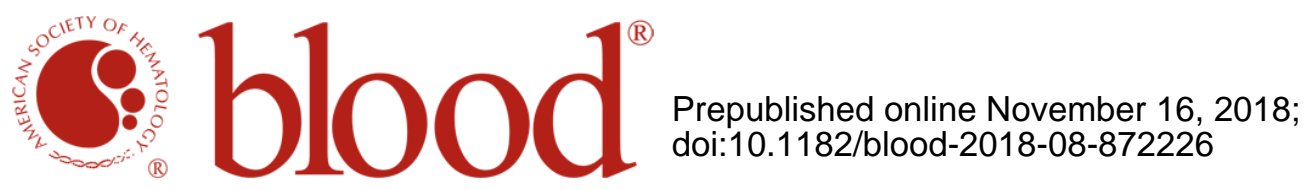

\section{The genomic landscape of plasma cells in systemic light chain amyloidosis}

Eileen M. Boyle, Cody Ashby, Christopher P. Wardell, Dorota Rowczenio, Sajitha Sachchithanantham, Yan Wang, Sarah K. Johnson, Michael A. Bauer, Niels Weinhold, Martin F. Kaiser, David C. Johnson, John R. Jones, Charlotte Pawlyn, Paula Proszek, Carolina Schinke, Thierry Facon, Charles Dumontet, Faith E. Davies, Gareth J. Morgan, Brian A. Walker and Ashutosh D. Wechalekar

Information about reproducing this article in parts or in its entirety may be found online at:

http://www.bloodjournal.org/site/misc/rights.xhtml\#repub_requests

Information about ordering reprints may be found online at:

http://www.bloodjournal.org/site/misc/rights.xhtml\#reprints

Information about subscriptions and ASH membership may be found online at:

http://www.bloodjournal.org/site/subscriptions/index.xhtml

Advance online articles have been peer reviewed and accepted for publication but have not yet appeared in the paper journal (edited, typeset versions may be posted when available prior to final publication). Advance online articles are citable and establish publication priority; they are indexed by PubMed from initial publication. Citations to Advance online articles must include digital object identifier (DOls) and date of initial publication.

Blood (print ISSN 0006-4971, online ISSN 1528-0020), is published weekly by the American Society of Hematology, 2021 L St, NW, Suite 900, Washington DC 20036.

Copyright 2011 by The American Society of Hematology; all rights reserved. 